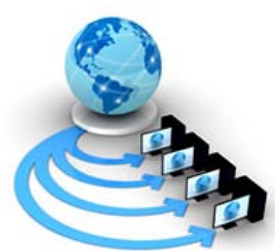

Volume 8, No. 9, November-December 2017

International Journal of Advanced Research in Computer Science

RESEARCH PAPER

Available Online at www.ijarcs.info

\title{
IMPLEMENTATION OF ENHANCED WEIGHT BASED CLUSTERING ROUTING PROTOCOL SCHEME (EWBCRPS) IN ADHOC NETWORK
}

\author{
Prabhdeep Kaur \\ Department of Computer Science \& Engg \\ Sri Sai College of Engg and Technology, Manawala (Amritsar) \\ Punjab (India)
}

\author{
Rimmy Chuchra \\ Department of Computer Science \& Engg \\ Sri Sai College of Engg and Technology, Manawala (Amritsar) \\ Punjab (India)
}

\begin{abstract}
In recent years, wireless systems are picking up prominence to its crest today, as the client needs wireless network independent of their geographical position. Wireless system is a system set up by utilizing radio sign recurrence to convey among PCs and other system gadgets. There are number of characteristics in mobile ad hoc networks, such as the dynamic network topology, limited bandwidth and energy constraint in the network. Mobile ad hoc network is useful for different purposes e.g. military operation to provide communicate between squads, emergency case in out-of-the-way places, medical control etc. Each mobile node acts as a host in the network when it receives the information. Efficiency of the network is the main concern in mobile ad hoc network to increase the network performance. There are many routing protocols in mobile ad hoc networks to increase the network performance. Also routing and efficiency of the network are the challenging issues in MANETs. Clustering is an efficient way to improve the network performance and routing techniques. In this research, Cluster based routing protocol is been used and analyzed the performance of cluster based routing protocol on NS-2 simulator tool with random number of nodes graphically. Cluster based routing protocol is been used for clustering in which cluster head is elected on the basis of minimum if of node. CBRP uses only a single parameter to elect a cluster head that is not a good way to evaluate the performance of network graphically. In MANETs, nodes are mobile so it is difficult to analyze the performance of network and also all these techniques has less efficiency. It reduces the network performance and reduces quality of service. Different techniques have their own drawbacks. In this work, further enhancement is implemented in cluster based routing protocol to change its cluster head scheme. In this scheme, the node is selected as a cluster head which has a minimum weight among the neighbor nodes. This technique is totally dynamic in nature. Every time when simulation starts different cluster head will be chosen. The weight of node is changed after every simulation. The weight is calculated by using four parameters like trust, number of nodes, mobility and transmission power. The routing is possible only through on demand route of the network, due to this network traffic is less so delay will be reduced and PDR is high. The technique has been implemented in Network Simulator version 2 (NS2) with different number of nodes. The graphical results show that this protocol technique performs better than CBRP in terms of throughput, delay and packet delivery ratio in the network.
\end{abstract}

Keywords: Adhoc Network, Weight Based Clustering routing protocol, quality of service, Transmission Power, Trust, Number of Nodes and Mobility, throughput.

\section{INTRODUCTION}

To handle or manage complex connections in wired network is a difficult task so it overcome this problem network professionals gave an idea of Mobile Adhoc Network [9] in which a network can be formed or deformed on the sky on demand basis[9]. Without having any maintenance of the physical servers on earth. The main reason of popularity of such type of network is this because of it covers a large spectrum of area and uses dynamic topology approach that further helps to collaborate that two different routing protocol approaches viz. proactive routing protocol scheme and Reactive Routing protocol Scheme [1]. Due to three main distinct or unique features of the Mobile Adhoc Network viz. self-configuration, Adaptability, Limited Power Supply and No pre-defined boundaries help us for achieving a better performance during the communication of mobile nodes in Mobile Adhoc Network[13][14]. If the choice is incorrect then network professionals can not give guarantee for producing efficient results in MANET [9]. In other words, you may say if users always prefer Mobile Adhoc Network [9] then network professionals again are not for sure for producing a correct output without choosing a best routing protocol scheme. As studied by the authors in their literature review, for gaining maximum performance the choice of routing protocol scheme is one of the mandatory or most important factor [2]. The increased growth of Adhoc network declares it is more efficient rather than other networks as seen the maximum use in our daily life practical applications as an example military battle field and commercial sector[15] etc. Obviously, these several types of practical applications may run on different types of routing protocol schemes as an example cluster based routing protocol scheme[1][12], dynamic source routing protocol scheme, AODV routing Protocol Scheme and weight based cluster routing protocol scheme[16].

In this research paper, Enhanced Weight based Cluster Routing protocol Scheme (EWBCRPS) is implemented. This scheme is work on the basis of three metrics and 4 parameters. These parameters can be calculated on the basis of weight. These three metrics are throughput, delay and packet delivery ratio and four different parameters are transmission power, high trust value, number of nodes and mobility etc. The number of nodes may vary in NS-2 Simulation Environment. The main motive to implement this new scheme is to reduce the amount of delay during packet transmission from the source to receiver end and improves throughput. Automatically, as the amount of delay during transmission of packets is decreases the packet delivery ratio is automatically increases. In this way, as the delay reduced and PDR (Packet delivery ratio) is automatically improved. And the major objective of this new designed scheme is 
achieved. Hence, user can achieve efficient results and the overall performance of the adhoc network is increases.

\section{REVIEW OF LITERATURE}

Omar Sail and Bernard Cousin et al. (2016):- This paper discusses about the energy conservation is a critical requirement for designing routing protocols for adhoc networks and correspondingly proposes a new methodology named energy efficient multi-path routing protocol which preserves residual energy of nodes and also helps to increase the life time of the network. Hence, this new designed methodology gives better performance in terms of energy conservation and end-to-end delay. [3]

M.V Rajesh and T.V.S Gireendranath et al. (2017): In this paper discusses about the architecture of MANET and correspondingly tells how the performance of the network is actually calculated by considering some different parameters of the network as an example delay, battery power and residual energy etc. Majorly, authors also evaluated about the novel cluster based algorithm with lesser energy consumption that gives better results than already existing algorithms. [4] Rakesh kumar and Piyush Verma et al. (2013): Authors discussed about the overall performance of the adhoc network by considering different-2 parameters by utilizing different routing protocols schemes. This paper also designed a new routing protocol scheme by utilizing Evolutionary algorithms (EA) and analytical hierarchy process for receiving optimized output of the MANET. [5]

Gautam M. Borkar and Anjali R.Mahajan et al. (April 2017): This paper shows the simulation numerical results of performance analysis that clearly describes that our provided proposed routing protocol generates better packet delivery ratio and decrease the amount of packet delay that automatically reduce the overhead of the secure environment.[6]

M. Anupama and Bachala Sathyanarayna et al. (December 2011): In this paper, authors compare some of the existing algorithms on clustering and categorize them on location based, weight based, and mobility based and then evaluate which clustering approach is best by considering some certain parameters.[7]

Mansi Sharma and Sanjay Kumar Dubey et al. (2014): Authors discusses about the main reason for applying clustering approach in MANET. This technique is more manageable and more viable. The working of this proposed scheme is based on the worthy node from the cluster as the cluster head is choose by applying some strategy and consider in such a way that helps to reduce the overhead and increase the overall efficiency of the routing protocol. [8]

S. K. Dhirendra et al. [2012] planned as well as executed a new strategy intended for inter as well as intra chaos courseplotting. This formula usually takes the advantage of practical as well as reactive course-plotting methods. Regarding inter as well as intra chaos course-plotting, practical as well as reactive aspects are widely-used respectively that's provided enhanced functionality intended for huge networks. We have got split the full circle into many chaos which has a chaos mind intended for chaos design as well as servicing. A chaos will be given by simply just one key factor that is the absolute maximum distance granted on the chaos mind. Each and every chaos mind preserves the a couple course-plotting kitchen tables. Aggressive course-plotting kitchen table intended for intra chaos as well as reactive course-plotting intended for inter chaos. Main factor is used to be able to break down the full circle in numerous overlapping subwoofer cpa networks. The true secret issue is dependent in total number regarding nodes within the circle. This benefit will be neither huge nor tiny. If it is tiny then chaos dimension can tiny. The number of groupings within the circle increases as well as reactive course-plotting expense will likely be increased. This method does not contemplate the very idea of expression dependent system. While just about any chaos node wishes to ahead facts packets to be able to friend chaos nodes, it mail a RREQ to be able to it is chaos head to transmit communication to be able to it is trip nodes. These gateways nodes ahead the packages for their friend chaos mind as well as solution quickly towards source's chaos mind node. Waiting around period as well as expense usually is lessened by simply this procedure simply because RREQ communication seriously isn't transmitting within the complete circle. [10]

B. Abdelhak et al. [2013] considered a new efficient weight based clustering algorithm. In this, each cluster is supervised by its cluster in order to ensure the acceptable level of security. It also improve the lifespan of mobile node in the network and reduces the energy consumption, decrease the total overhead and maintaining the stable cluster [11].

\section{RESEARCH DESIGN}

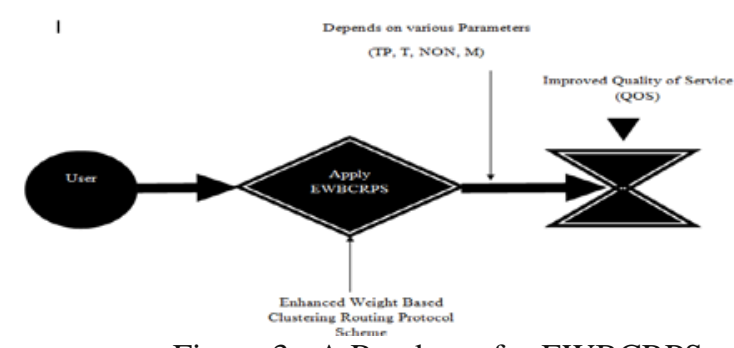

Figure 3: A Roadmap for EWBCRPS.

Table 1:- Nomenclature of Parameters Dependency on OWBCRPS.

\begin{tabular}{|c|c|c|}
\hline S.No & List of Parameters Dependent & Abbreviations \\
\hline 1 & TP & Transmission Power \\
\hline 2 & T & Throughput \\
\hline 3 & NON & Number of Nodes \\
\hline 4 & M & Mobility \\
\hline
\end{tabular}

\section{GRAPHICAL REPRESENTATION OF IMPLEMENTED EWBCRPS (ENHANCED WEIGHT BASED CLUSTERING ROUTING PROTOCOL SCHEME)}

\subsection{Graphical representation of Throughput when run} EWBCRPS: 


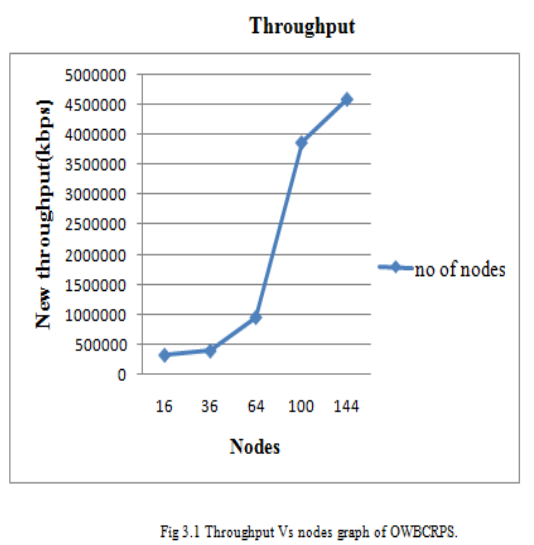

This graphical representation of throughput 3.1 shows the steady increase in Throughput curve by increasing no of nodes which shows the improved quality of service of EWBCRPS.

\subsection{Graphical representation of Delay when run OWBCRPS:}

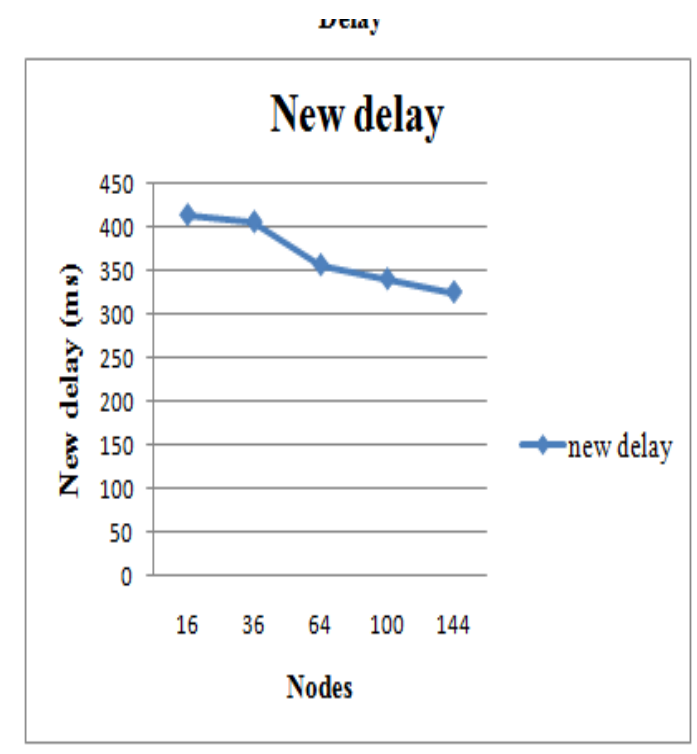

Fig 3.2 Delay Vs nodes graph of OWBCRPS.

This graphical representation of throughput 3.2 by increasing nodes the delay parameters is going to be decreased when run on EWBCRPS. Alternatively, the packet sending \& receiving ratio on source and destination path is going to be increased.

\subsection{Graphical representation of PDR (Packet Delivery Ratio) when run EWBCRPS:}

PDR

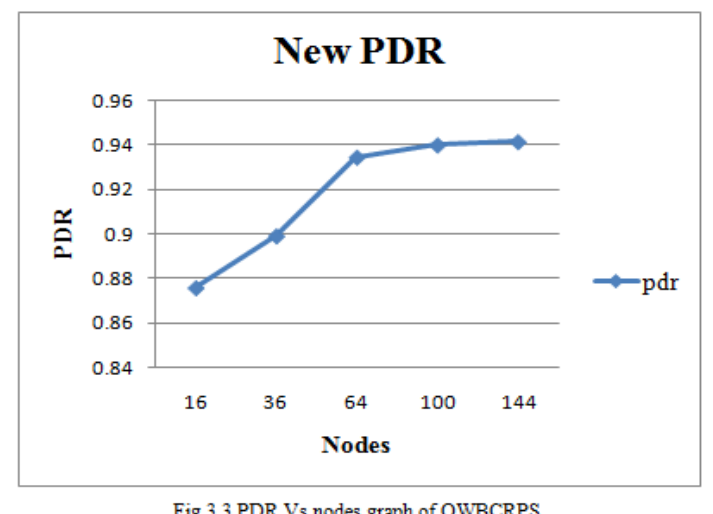

As shown in the graphical representation of 3.2 Delay case, as this delay factor is going to be decreased when PDR in run graph 3.3 new scheme of EWBCRPS then automatically the packet delivery ratio(PDR) is increased.

Hence, these above given graphical representations shows these three metrics ( Throughput, Delay and PDR) when run on new scheme "EWBCRPS" named Optimized Weight Based Clustering Routing protocol Scheme on NS2 Simulator then the throughput, packet delivery ratio is going to be increased and the delay parameter is going to be decreased. These three metrics can be calculated on the basis of 4 different parameters (Transmission Power, High Trust rate, Number of nodes and Mobility factor). In other words, authors say by considering 3 different metrics depend on above given 4 parameters the overall performance of the adhoc network can be easily enhanced that automatically helps to increase the quality of service.

\section{CONCLUSION}

Different types of routing protocols are used in mobile ad hoc networks. MANET is basically a type of multi-hop wireless network that is used for establishing dynamic link without any pre -existing infrastructure. This research is based on the simulation of enhanced CBRP (Cluster Based Routing Protocol) model with varying number of nodes that uses varying mobility as well using NS 2 Simulator. In my thesis research, I modified the cluster head election scheme of weight based routing protocol "EWBCRP" is termed as Enhanced Weight Based Clustering Routing Protocol by considering four different parameters i.e. trust, number of nodes, mobility and transmission power that ultimately helps to improve Quality of Service(QOS). While calculating these four parameters, the performance of the Optimized/Enhanced weight based cluster routing protocol can be easily improved and after this I performed the comparative study of the existing scheme and the proposed scheme. The performance of the new designed scheme is greater than the existing scheme as shown in the above given graphs based on different parameters. This comparative analysis shows their dynamic behavior and its simulation results shows their better quality of service (QOS) depends on some specific parameters. Hence, the comparative analysis shows that the performance of the Mobile Adhoc Network is enhanced by considering some important factors as an example 
transmission power, trust, varying number of nodes and mobility factor.

\section{FUTURE SCOPE}

In future, this work can be extended by changing the routing protocol scheme by increasing number of nodes in the ad hoc network. This idea may facilitate to reduce the amount of delay of data packets as well as response time that automatically increase the throughput as well as overall performance of the ad hoc network.

\section{REFERENCES}

[1] Jinke Huang and Xiaoguang Fan, "A Clustering Routing protocol for Mobile Adhoc Network”, Mathematical Problems in Engineering”, 2016, China.

[2] Sunil Pathak and Sonal Jain,” A Novel Weight based Clustering algorithm for Routing in MANET”, Vol.22, No.8, Nov 2016, Springer-Verleg, New York.

[3] Omar Sail and Bernard Cousin,” Energy Aware and Stable Cluster Based Multipath Routing Protocol for Wireless Adhoc Network", International Journal of Networking and Virtual Organizations, 2016, Algeria.

[4] M.V Rajesh and T.V.S Gireendranath,” A Novel Energy Efficient Cluster Based Routing Protocol for Highly Dense MANET Architecture", International Journal of Computational Intelligence Research, Vol.13, No.5, 2017.

[5] Rakesh kumar and Piyush Verma," Mobile Adhoc Network and its Routing protocols", World Academy of Science,Engineeing \& technology- International Journal of Computer and Information Engineering,Vol.7,No.8,2013.

[6] Gautam M. Borkar and Anjali R.Mahajan, "Secure Routing Environment with Enhancing Quality of Service in MANET", International Journal on recent and Innovation Trends in Computing and Communication, Vol.5, No.4, April 2017.
[7] M. Anupama and Bachala Sathyanarayna," Survey of Cluster Based Routing Protocols in MANET", International Journal of Computer theory and Engineering, Vol.3, No.6, December 2011.

[8] Mansi Sharma and Sanjay Kumar Dubey,” Analytical Study of CBRP in Mobile Adhoc Network", International Journal of Computer and Communication System Engineering, 2014.

[9] Manpreet Kaur, Sarabjeet Kaur, Anshu Sharma et al. "An Optimized Weight Based Clustering Routing Protocol Scheme In MANETS For Improved QOS”, International Journal of Engineering Applied Sciences and Technology, Vol. 1, Issue 1, June 2016.

[10] S. K. Dhirendra, K. Chiranjeev, “An Efficient Cluster Based Routing Protocol for MANET”, 3rd IEEE International Advance Computing Conference (IACC), pp: 224-229, 2012.

[11] B. Abdelhak, H. Saad, B. Abdelhak, "A Weight Based Clustering Scheme for Mobile Adhoc Networks", ACM Vienna, Austria, 2013.

[12] R. Balasubramaniyan et al. "An improved cluster based routing protocol with backup cluster head for MANETs", ARPN journal of engineering and applied sciences, Vol. 10 pp: 4927-4930, 2015.

[13] R. Rajesh, Dr A. Saradha, "Efficient MAC Based AODV Routing Protocol to improve the efficiency of MANET", Journal of Computer Science and Engineering, Vol. 2 No. 6, pp: 37-48, 2015.

[14] S. Rajesh, S. S. Barde, "Survey on Cluster Based Routing Protocol in MANETs", International Journal of Science and Research, Vol. 4, No. 1, 2015.

[15] C. Naveen et al. "A Distributed weighted cluster based routing protocol for MANETs”, Springer- Verlag Berlin Heidelberg, pp 147-151, 2011.

[16] Prabhdeep Kaur and Rimmy Chuchra” A Novel Weight Based Clustering Routing Protocol Scheme for Improved QOS in Adhoc Network”, International Journal of Advance Research in Computer Science, Vol.8, No.7, July-August 2017. 